\title{
Análise do tempo para o alívio da intensidade da dor em pacientes com dor lombar crônica não específica via modelo de riscos proporcionais de Cox
}

Analysis of the time to relieving pain in patients with chronic non-specific low back pain via Cox proportional hazard model

Jéssica Ferreira Silva ${ }^{1}$, Richard Eloin Liebano ${ }^{2}$, Juliana Barbosa Corrêa ${ }^{2}$, Raul Yukihiro Matsushita ${ }^{1}$ e Eduardo Yoshio Nakano ${ }^{1}$

${ }^{1}$ Departamento de Estatística, Universidade de Brasília, Brasília/DF, Brasil jessiicafsilva@gmail.com; raulmta@unb.br;nakano@unb.br

${ }^{2}$ Departamento de Fisioterapia, Universidade Federal de São Carlos, São Carlos/SP, Brasil liebano@gmail.com; julianabcorrea@gmail.com

\section{Resumo}

O objetivo do presente estudo foi avaliar o tempo até o alívio da intensidade da dor em pacientes com dor lombar crônica não específica tratados com corrente interferencial. O conhecimento do número de sessões necessárias para o alivio da intensidade da dor é de grande importância, pois pode ser utilizada como medida de efetividade do tratamento, além de auxiliar o fisioterapeuta na escolha do melhor tratamento a ser utilizado. A análise foi realizada em uma amostra de 150 pacientes com dor lombar crônica não específica, utilizando o modelo de riscos proporcionais de Cox. Toda a análise foi realizada pelo software livre $R$.

Palavras-chave: Análise de Sobrevivência; dados censurados; eletroestimulação; dor lombar

\begin{abstract}
The aim of this study was to evaluate the time to relieving pain intensity in patients with non-specific chronic low back pain treated by interferential current. Knowledge of the number of sessions needed for relieving pain intensity is very important because it can be used as a measure of treatment effectiveness, and assists the physiotherapist in choosing the best treatment to be used. The analysis was conducted on a sample of 150 patients with non-specific chronic low back pain, using the Cox proportional hazard model. All analysis were performed by the free software $R$.
\end{abstract}

Keywords: Survival analysis; censored data; electrical simulation; low back pain 


\section{Introdução}

A dor lombar é caracterizada por dor e desconforto localizado abaixo da margem costal e acima da dobra glútea inferior, com ou sem dor referida para membros inferiores (AIRAKSINEN et. al, 2006). Trata-se de um importante problema de saúde pública na sociedade moderna, podendo interferir diretamente na qualidade de vida e afetar as atividades de vida diária (AVD’s) (DELITTO et. al, 2012), sendo também responsável por altos índices de absenteísmo no trabalho, além de altos custos socioeconômicos mundialmente (DELITTO et al., 2012; TULDER, 2006).

O uso de exercícios consiste em uma das maiores evidências para o tratamento da dor lombar crônica (AIRAKSINEN et al, 2006; COSTA et al, 2009). Entretanto, pacientes com dor lombar podem apresentar dor importante, que leva à limitação da capacidade física, dificultando a execução de exercícios. Assim, o uso de agentes eletrofísicos para alívio da dor poderia tornar esses pacientes aptos a participar de um programa de exercícios. Dentre os recursos eletrofísicos, a corrente interferencial (CI) vem sendo investigada para o tratamento da dor lombar (FACCI et al, 2011; FUENTES et al, 2010).

Neste contexto, o objetivo geral deste trabalho foi ajustar o modelo de riscos proporcionais de Cox (COX, 1972) para modelar o tempo até o alívio da intensidade da dor em pacientes com dor lombar crônica não específica. $O$ evento de interesse (alívio da intensidade da dor) é definido como o número de sessões que cada paciente foi submetido até ser observado o evento de interesse, que é a diminuição ou alívio da intensidade da dor. O alívio da dor foi caracterizado como a redução em no mínimo $50 \%$ da escala numérica verbal de dor, em relação ao valor observado no início do tratamento. $\mathrm{O}$ estudo foi realizado dentro do contexto de análise de sobrevivência, utilizando o modelo de riscos proporcionais de Cox. Toda a análise foi realizada através da biblioteca survival do software livre R, versão 3.3.1 (R CORE TEAM, 2016).

\section{Material e método}

Este estudo foi uma análise secundária dos dados de um estudo anterior comparando corrente interferencial ativa com corrente interferencial placebo em 150 pacientes com dor lombar crônica (CORRÊA et. al, 2016).

\subsection{Participantes}

Foram incluídos no estudo indivíduos com idades entre 18 e 80 anos e de ambos os gêneros que apresentavam quadro de dor lombar não específica há pelo menos 3 meses e obtiveram nível mínimo de dor 3 na escala verbal numérica da dor nos últimos 7 dias. Foram excluídos pacientes portadores de doenças graves de coluna, tais como fraturas, tumores e doenças inflamatórias, como espondilite anquilosante; condições radiculares da coluna confirmadas por testes neurológicos (hérnia discal e espondilolistese com comprometimento neurológico, estenose de canal medular e outros); doenças neurológicas; doenças cardiorrespiratórias graves; gravidez; infecção no local de aplicação da corrente; câncer; marcapasso cardíaco; lesões de pele no local de aplicação da corrente; alterações de sensibilidade e alergia na região de posicionamento dos eletrodos.

Os participantes foram avaliados pelo terapeuta responsável pelas avaliações durante a pesquisa para verificação de enquadramento nos critérios de inclusão do estudo. Caso fossem elegíveis, os participantes eram 
informados sobre os objetivos do estudo. A seguir, foram orientados a assinar um Termo de Consentimento Livre e Esclarecido (TCLE) para participação no estudo.

\subsection{Randomização}

A alocação dos sujeitos foi feita de forma aleatória, utilizando o programa radomization.com na proporção de 2:1 e os códigos dos grupos foram guardados em envelopes lacrados e opacos.

\subsection{Intervenção}

Os participantes foram distribuídos aleatoriamente em 2 grupos: Grupo Ativo $(n=100)$ e Grupo Placebo $(n=50)$. Todos os participantes receberam 30 minutos de estimulação com a corrente (placebo com corrente desligada), 3 vezes por semana em dias alternados, por 4 semanas, totalizando 12 sessões.

No estudo foi utilizado o equipamento gerador de correntes alternadas de média frequência (Neurovector) da Indústria Brasileira de Equipamentos Médicos (IBRAMED) ${ }^{\circledR}$. A técnica utilizada foi o modo bipolar com 2 canais a 5 cm do processo espinhoso de L3 e L5 (Figura 1). Os seguintes parâmetros foram utilizados: frequência da corrente portadora de 1 e $4 \mathrm{kHz} ; \mathrm{AMF}=100 \mathrm{~Hz} ; \Delta \mathrm{F}=50 \mathrm{~Hz}$; swing pattern de 1:1 e 30 minutos de estimulação.

O Grupo Placebo recebeu os procedimentos semelhantes aos demais grupos, porém a amplitude da corrente não foi aumentada. A cada 5 minutos o terapeuta perguntava ao participante se a sensação de "formigamento forte, porém confortável" se mantinha. No caso de diminuição da sensação da corrente, a amplitude da corrente era aumentada até que o participante alcançasse a sensação anterior.

\subsection{Instrumento de medida}

A intensidade da dor foi avaliada através da escala verbal numérica da dor, que avalia os níveis de intensidade da dor percebida pelo paciente por meio de uma escala de 11 pontos (variando de 0 a 10), sendo 0 classificado como "sem dor alguma" e 10 "a pior dor que poderia sentir". A avaliação da dor foi realizada de forma verbal, na qual o paciente relatou a intensidade da dor. Neste trabalho, a variável resposta é o número de sessões necessárias até o alívio ou a diminuição da dor. O alívio da dor foi caracterizado como a redução em no mínimo $50 \%$ da escala numérica de dor em relação ao valor observado no início do tratamento.

\subsection{Consumo de analgésicos}

Para avaliar o consumo de analgésicos durante o tratamento, o avaliador preencheu um recordatório com os dias da semana do início do tratamento até a data da última sessão para marcação do uso de analgésicos ou antiinflamatórios e a dosagem dos mesmos durante o tratamento.

\subsection{Covariáveis}

As covariáveis consideradas foram sexo, idade, Índice de Massa Corpórea (IMC), tempo de dor, uso de medicamentos e grupo de tratamento. A variável idade foi categorizada de acordo com seus quantis e seus níveis foram agrupados à medida que apresentaram riscos relativos individuais semelhantes. A variável IMC foi dicotomizada classificando os pacientes em obesos, que apresentaram IMC $\geq 30$ e não obesos, que apresentaram IMC $<30$. A variável tempo de dor foi dicotomizada discriminando os pacientes com até um ano de dor com aqueles com pelo menos um ano de dor. A amostra final foi composta por 150 pacientes, no qual 9 (6\%) foram observações censuradas. A distribuição de frequências das categorias das seis covariáveis é apresentada na Tabela 1. 
Tabela 1 - Frequências e percentuais das covariáveis consideradas no estudo

\begin{tabular}{|c|c|c|c|}
\hline & Variável & $\mathbf{N}$ & $\%$ \\
\hline \multicolumn{4}{|l|}{ Sexo } \\
\hline & Masculino & 35 & $23,3 \%$ \\
\hline & Feminino & 115 & $76,7 \%$ \\
\hline \multicolumn{4}{|l|}{ Idade } \\
\hline & Até 50 anos & 66 & $44,0 \%$ \\
\hline & 50 anos ou mais & 84 & $56,0 \%$ \\
\hline \multicolumn{4}{|l|}{ IMC } \\
\hline & 30 ou mais (obeso) & 108 & $72,0 \%$ \\
\hline & Até 30 (não obeso) & 42 & $28,0 \%$ \\
\hline \multicolumn{4}{|c|}{ Tempo de dor } \\
\hline & 5 anos ou mais & 93 & $62,0 \%$ \\
\hline & Menos de 5 anos & 57 & $38,0 \%$ \\
\hline \multicolumn{4}{|c|}{ Uso de medicamentos } \\
\hline & Sim & 115 & $76,7 \%$ \\
\hline & Não & 35 & $23,3 \%$ \\
\hline \multicolumn{4}{|c|}{ Grupo de tratamento } \\
\hline & Grupo Placebo & 50 & $33,3 \%$ \\
\hline & Grupo Ativo & 100 & $66,7 \%$ \\
\hline
\end{tabular}

\subsection{Modelo estatístico}

Os dados do tempo até o alívio da dor foram analisados por meio de técnicas de Análise de Sobrevivência. O tempo até o alívio da dor foi representado por uma variável discreta, representando o número de sessões necessárias até a diminuição ou alívio da dor. A observação foi considerada censurada à direita em situações nas quais, por algum motivo, foi perdido o acompanhamento do paciente durante o período de estudo ou o paciente não percebeu um alívio na dor após as 12 sessões de tratamento realizadas. A análise foi realizada considerando o modelo semi-paramétrico de riscos proporcionais de Cox (COX, 1972). Este modelo propõe modelar os dados de sobrevivência, na presença de covariáveis por meio da função de risco. Segundo o modelo de Cox, a função de risco para um indivíduo com vetor de covariáveis $\mathbf{x}$ é dada por

$$
h_{\mathrm{Cox}}(t \mid \mathbf{x})=h_{0}(t) \exp \left\{\mathbf{x}^{\prime} \boldsymbol{\beta}\right\},
$$

em que $h_{0}(t)$ é conhecida como função de risco base, ou seja, o risco de um indivíduo com todas as covariáveis iguais a zero e $\boldsymbol{\beta}$ é o vetor de coeficientes de regressão desconhecidos.

A função de sobrevivência para um indivíduo com vetor de covariáveis $\mathbf{x}$ está relacionada com a função de risco (1) e pode ser escrita como

$$
S_{\mathrm{Cox}}(t \mid \mathbf{x})=\left[S_{0}(t)\right]^{\exp \left\{\mathbf{x}^{\prime} \boldsymbol{\beta}\right\}}
$$

Aqui, $S_{0}(t)$ é a função de sobrevivência base, ou seja, a função de sobrevivência de um indivíduo com todas as covariáveis iguais a zero. Trabalhos que apresentam aplicações do modelo de riscos proporcionais de Cox podem ser vistos em Nakano e Cunha (2012), Santos e Nakano (2015), Maia e Nakano (2016), entre outros. Inicialmente, um 
modelo de riscos proporcionais de Cox simples foi ajustado para cada uma das 6 covariáveis separadamente. A suposição de proporcionalidade dos riscos foi verificada por meio dos resíduos escalonados de Schoenfeld, que são centralizados no zero e são independentes do tempo se os riscos são proporcionais (SCHOENFELD, 1982). Ao final, um modelo de riscos proporcionais de Cox múltiplo foi ajustado considerando todas as covariáveis que não violaram a suposição de proporcionalidade da função de risco. O ajuste global do modelo final foi verificado pelo resíduo de CoxSnell (COX e SNELL, 1968) e um possível problema de multicolinearidade foi verificado por meio do FIV - Fator Inflacionário da Variância (NETER et al., 1990).

\section{Resultados e discussão}

O modelo de riscos proporcionais de Cox foi ajustado para os dados do tempo até o alívio da intensidade da dor. A verificação da suposição básica do modelo de Cox, que assume proporcionalidade dos riscos, foi avaliada por meio de um teste qui-quadrado do coeficiente de correlação entre o tempo de sobrevivência e o resíduo escalonado de Schoenfeld (GRAMBSCH e THERNEAU, 1994). A Tabela 2 apresenta o ajuste do modelo de Cox para cada uma das 6 covariáveis separadamente, assim como o teste de tendências do resíduo de Schoenfeld. Pode-se observar pela Tabela 2 que, das seis variáveis analisadas, somente o grupo de tratamento $(p=0,003)$ e uso de medicamentos $(p=0,010)$ apresentaram resultados significativos. Esse resultado indica que os pacientes do Grupo Ativo precisaram de um número menor de sessões para o alívio da dor, quando comparado com o Grupo Placebo e que o alívio da intensidade da dor foi mais rápido para aqueles pacientes que não usam medicamentos. A Figura 1 apresenta as estimativas de Kaplan-Meier (KAPLAN e MEIER, 1958) da função de sobrevivência do tempo até o alívio da dor segundo as covariáveis consideradas no estudo.

Tabela 2 - Estimativas dos parâmetros do modelo de riscos proporcionais de Cox simples e valor p do teste de tendências do resíduo de Schoenfeld

\begin{tabular}{|c|c|c|c|c|c|}
\hline \multirow[b]{2}{*}{ Variável $^{(2)}$} & & \multicolumn{3}{|c|}{ Ajuste do modelo simples ${ }^{(1)}$} & \multirow{2}{*}{$\begin{array}{c}\begin{array}{c}\text { Teste de } \\
\text { tendências }\end{array} \\
p^{(5)}\end{array}$} \\
\hline & & $\beta$ (EP) & $\mathrm{RR}^{(3)}(\mathrm{IC} 95 \%)$ & $p^{(4)}$ & \\
\hline \multicolumn{6}{|l|}{ Sexo } \\
\hline & Masculino & 0 & 1 & & \\
\hline & Feminino & $0,056(0,199)$ & $1,058(0,716-1,562)$ & 0,777 & 0,845 \\
\hline \multicolumn{6}{|l|}{ Idade } \\
\hline & Até 50 anos & 0 & 1 & & \\
\hline & 50 anos ou mais & $0,169(0,169)$ & $1,184(0,851-1,649)$ & 0,317 & 0,583 \\
\hline \multicolumn{6}{|l|}{ IMC } \\
\hline & 30 ou mais (obeso) & 0 & 1 & & \\
\hline & Até 30 (não obeso) & $-0,010(0,186)$ & $0,990(0,688-1,424)$ & 0,956 & 0,832 \\
\hline \multicolumn{6}{|c|}{ Tempo de dor } \\
\hline & 5 anos ou mais & 0 & 1 & & \\
\hline & Menos de 5 anos & $0,223(0,174)$ & $1,250(0,889-1,757)$ & 0,200 & 0,064 \\
\hline \multicolumn{6}{|c|}{ Uso de medicamentos } \\
\hline & Sim & 0 & 1 & & \\
\hline & Não & $0,507(0,199)$ & $1,660(1,126-2,446)$ & 0,010 & 0,819 \\
\hline \multicolumn{6}{|c|}{ Grupo de tratamento } \\
\hline & Grupo Placebo & 0 & 1 & & \\
\hline & Grupo Ativo & $0,553(0,185)$ & $1,738(1,209-2,499)$ & 0,003 & 0,859 \\
\hline
\end{tabular}

(1) Modelo de Cox ajustado para cada variável separadamente. ${ }^{(2)}$ As categorias com coeficientes $\beta$ iguais a zero são os níveis de referência das variáveis; ${ }^{(3)} \mathrm{RR}=$ Risco Relativo; ${ }^{(4)}$ Valor $\mathrm{p}$ do teste de significância do coeficiente do modelo de Cox simples; ${ }^{(5)}$ Valor $\mathrm{p}$ do teste de significância coeficiente de correlação entre o tempo de sobrevivência e o resíduo de Schoenfeld. 


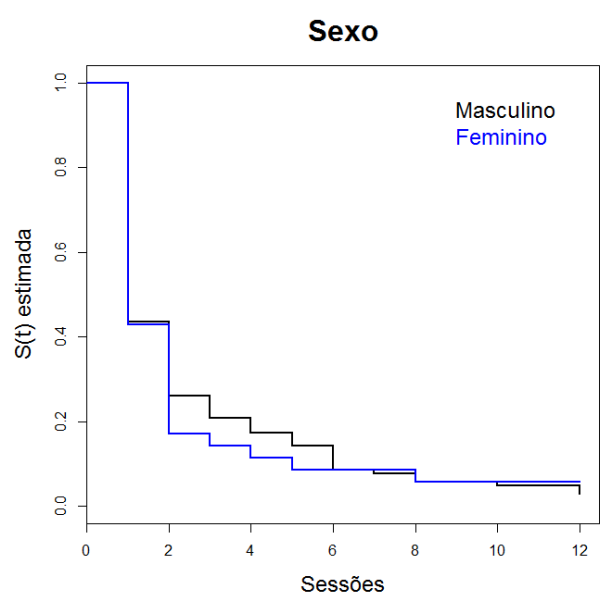

IMC
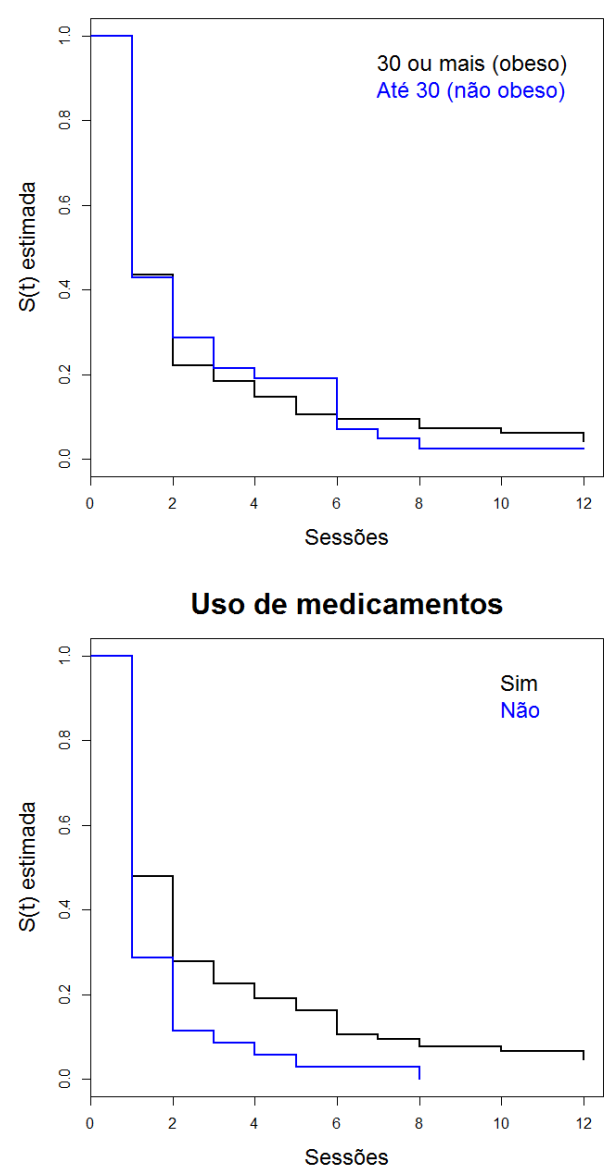

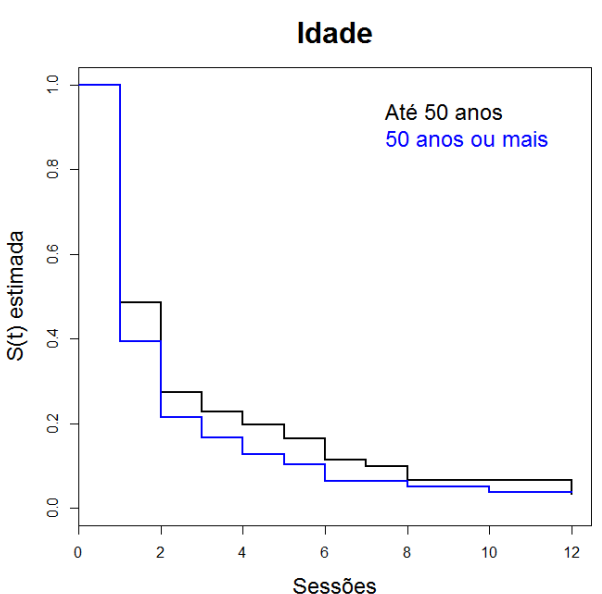

Tempo de dor
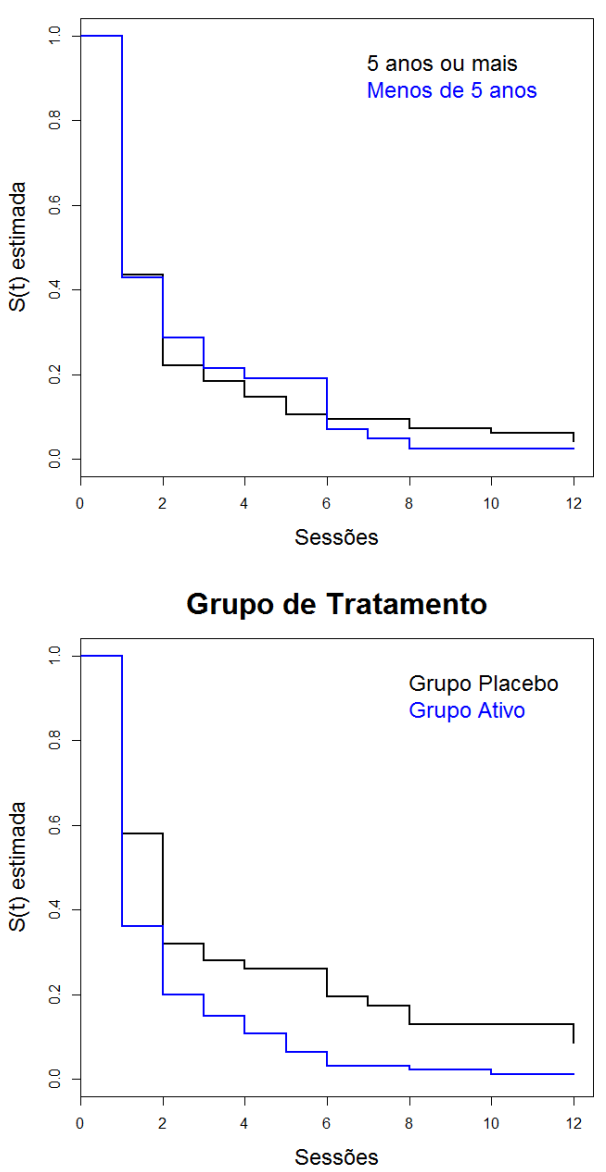

Figura 1 - Estimativas da função de sobrevivência via Kaplan-Meier segundo as covariáveis consideradas no estudo

Apesar de não terem apresentado resultados significativos $(p>0,05)$, as variáveis sexo, idade, IMC e tempo de dor foram mantidas no modelo múltiplo por se acreditar que são variáveis que, na prática, podem influenciar no resultado do tratamento. O teste de tendências não rejeitou a suposição de proporcionalidade da função de risco $(p>0,05)$ nas 6 variáveis estudadas. A Tabela 3 apresenta o ajuste do modelo de Cox múltiplo para as variáveis sexo, idade, IMC, tempo de dor, uso de medicamentos e grupo de tratamento, assim como o FIV - Fator Inflacionário da Variância. Pode-se observar, pelos resultados da Tabela 3, que as variáveis sexo, idade, IMC e tempo de dor não influenciaram de forma significativa o tempo até o alívio da dor $(p>0,05)$, sendo as variáveis grupo de tratamento $(p=0,006)$ e uso de medicamentos $(p=0,049)$ as únicas variáveis que apresentaram efeito significativo. Conforme os 
valores observados do FIV (próximo de 1 para todas as variáveis), não foram identificados problemas de multicolinearidade entre as variáveis, fato que também pode ser percebido devido à pequena variação entre os resultados obtidos pelo modelo simples e múltiplo.

Tabela 3 - Estimativas dos parâmetros do modelo de riscos proporcionais de Cox múltiplo e do Fator Inflacionário da Variância (FIV)

\begin{tabular}{|c|c|c|c|c|c|}
\hline \multirow[b]{2}{*}{ Variável $^{(2)}$} & & \multicolumn{3}{|c|}{ Ajuste do modelo múltiplo ${ }^{(1)}$} & \multirow[b]{2}{*}{ FIV } \\
\hline & & $\beta$ (EP) & $\operatorname{RR}^{(3)}(\mathrm{IC} 95 \%)$ & $p^{(4)}$ & \\
\hline \multicolumn{6}{|l|}{ Sexo } \\
\hline & Masculino & 0 & 1 & & \\
\hline & Feminino & $0,013(0,206)$ & $1,013(0,677-1,516)$ & 0,949 & 1,035 \\
\hline \multicolumn{6}{|l|}{ Idade } \\
\hline & Até 50 anos & 0 & 1 & & \\
\hline & 50 anos ou mais & $0,122(0,178)$ & $1,129(0,797-1,601)$ & 0,494 & 1,082 \\
\hline \multicolumn{6}{|l|}{ IMC } \\
\hline & 30 ou mais (obeso) & 0 & 1 & & \\
\hline & Até 30 (não obeso) & $0,070(0,193)$ & $1,072(0,735-1,564)$ & 0,718 & 1,035 \\
\hline \multicolumn{6}{|l|}{ Tempo de dor } \\
\hline & 5 anos ou mais & 0 & 1 & & \\
\hline & Menos de 5 anos & $0,239(0,185)$ & $1,270(0,885-1,824)$ & 0,195 & 1,053 \\
\hline \multicolumn{6}{|c|}{ Uso de medicamentos } \\
\hline & Sim & 0 & 1 & & \\
\hline & Não & $0,408(0,207)$ & $1,503(1,001-2,258)$ & 0,049 & 1,044 \\
\hline \multicolumn{6}{|c|}{ Grupo de tratamento } \\
\hline & Grupo Placebo & 0 & 1 & & \\
\hline & Grupo Ativo & $0,516(0,191)$ & $1,676(1,152-2,438)$ & 0,007 & 1,030 \\
\hline
\end{tabular}

(1) Modelo de Cox ajustado para todas as variáveis; ${ }^{(2)}$ As categorias com coeficientes $\beta$ iguais a zero são os níveis de referência das variáveis; ${ }^{(3)}$ $\mathrm{RR}=$ Risco Relativo, considerando fixa as demais covariáveis. ${ }^{(4)}$ Valor $\mathrm{p}$ do teste de significância do coeficiente do modelo de Cox.

Assim, de acordo com o modelo de Cox múltiplo, o risco de alívio da intensidade da dor de um paciente tratado com corrente interferencial é 1,676 vezes (67,6\% maior) que o risco de alívio da dor de pacientes do grupo tratado com corrente interferencial placebo. Segundo o modelo de Cox (COX, 1972), a função de sobrevivência (2) para um indivíduo com vetor de covariáveis $\mathbf{x}$, é estimada por:

$$
\hat{S}_{\mathrm{Cox}}(t \mid \mathbf{x})=\left[\hat{S}_{0}(t)\right]^{\exp \left\{\mathbf{x}^{\prime} \hat{\boldsymbol{\beta}}\right\}}
$$

Aqui, $\hat{\boldsymbol{\beta}}$ é o vetor de parâmetros estimados do modelo de Cox múltiplo, cujas estimativas são apresentadas pela Tabela 3 e $\hat{S}_{0}(t)$ é estimativa da função de sobrevivência base (função de sobrevivência de um indivíduo com todas as covariáveis iguais a zero), cujas estimativas são apresentadas pela Tabela 4.

Tabela 4 - Estimativas da função de sobrevivência base

\begin{tabular}{cccc}
\hline Sessão & $\hat{S}_{0}(t)$ & Sessão & $\hat{S}_{0}(t)$ \\
\hline 1 & 0,6445 & 7 & 0,2270 \\
2 & 0,4634 & 8 & 0,1819 \\
3 & 0,4092 & 9 & 0,1819 \\
4 & 0,3627 & 10 & 0,1653 \\
5 & 0,3188 & 11 & 0,1653 \\
6 & 0,2413 & 12 & 0,1284 \\
\hline
\end{tabular}


Assim, de acordo com a função de sobrevivência estimada (3), tem-se, por exemplo, que a probabilidade de um paciente do sexo masculino, com 50 anos ou mais, com IMC menor que 30, com dor lombar há pelo menos 5 anos, que usa medicamentos e que está sendo tratado com corrente interferencial ativa não ter sua dor lombar aliviada em 3 sessões do tratamento é igual a:

$$
\begin{aligned}
\hat{S}_{\text {Cox }}(3 \mid \mathbf{x}) & =\left[\hat{S}_{0}(3)\right]^{\exp \{0+0,122+0,070+0+0+0,516\}} \\
& =0,4092^{\exp \{0,708\}} \\
& =0,5312
\end{aligned}
$$

Em contrapartida, a probabilidade de um paciente com mesmo perfil que está sendo tratado com corrente interferencial placebo não ter sua dor lombar aliviada em 3 sessões do tratamento é igual a:

$$
\begin{aligned}
\hat{S}_{\text {Cox }}(3 \mid \mathbf{x}) & =\left[\hat{S}_{0}(3)\right]^{\exp \{0+0,122+0,070+0+0+0\}} \\
& =0,4092^{\exp \{0,192\}} \\
& =0,8423
\end{aligned}
$$

A suposição de proporcionalidade dos riscos no modelo de Cox múltiplo foi avaliada pelo teste de tendências realizado a partir do coeficiente de correlação entre o tempo de sobrevivência e o resíduo escalonado de Schoenfeld. Tabela 5 apresenta os resultados do teste de tendências da função de risco para as seis covariáveis consideradas no modelo de Cox múltiplo.

Tabela 5 - Testes de tendências do resíduo de Schoenfeld

\begin{tabular}{lccc}
\hline Variável & $\boldsymbol{\rho}$ & $\chi^{\mathbf{2}}$ & $\boldsymbol{p}$ \\
\hline Sexo & $-0,0167$ & 0,0433 & 0,835 \\
Idade & $-0,0196$ & 0,0582 & 0,809 \\
IMC & 0,0044 & 0,0029 & 0,957 \\
Tempo de dor & 0,1451 & 3,3633 & 0,067 \\
Medicamentos & $-0,0180$ & 0,0505 & 0,822 \\
Tratamento & 0,0388 & 0,2349 & 0,628 \\
\hline GLOBAL & NA & 4,0462 & 0,6704 \\
\hline
\end{tabular}

Os resultados apresentados pela Tabela 5 indicam que não há tendências significativas, tanto para as seis variáveis consideradas quanto para o modelo global. Assim, a suposição de proporcionalidade dos riscos não está sendo violada na análise desses dados.

A verificação da qualidade do ajuste dos riscos proporcionais de Cox foi realizada por meio do resíduo de CoxSnell (COX e SNELL, 1968). Segundo Lawless (2003), os resíduos de Cox-Snell vêm de uma população homogênea e devem seguir uma distribuição exponencial com média 1. Quanto mais a função de sobrevivência dos resíduos do modelo de Cox se aproxima da função de sobrevivência da exponencial, melhor é o ajuste do modelo de Cox. A Figura 2 apresenta as comparações da estimativa de Kaplan-Meier da função de sobrevivência dos resíduos de Cox-Snell com a exponencial padrão. 

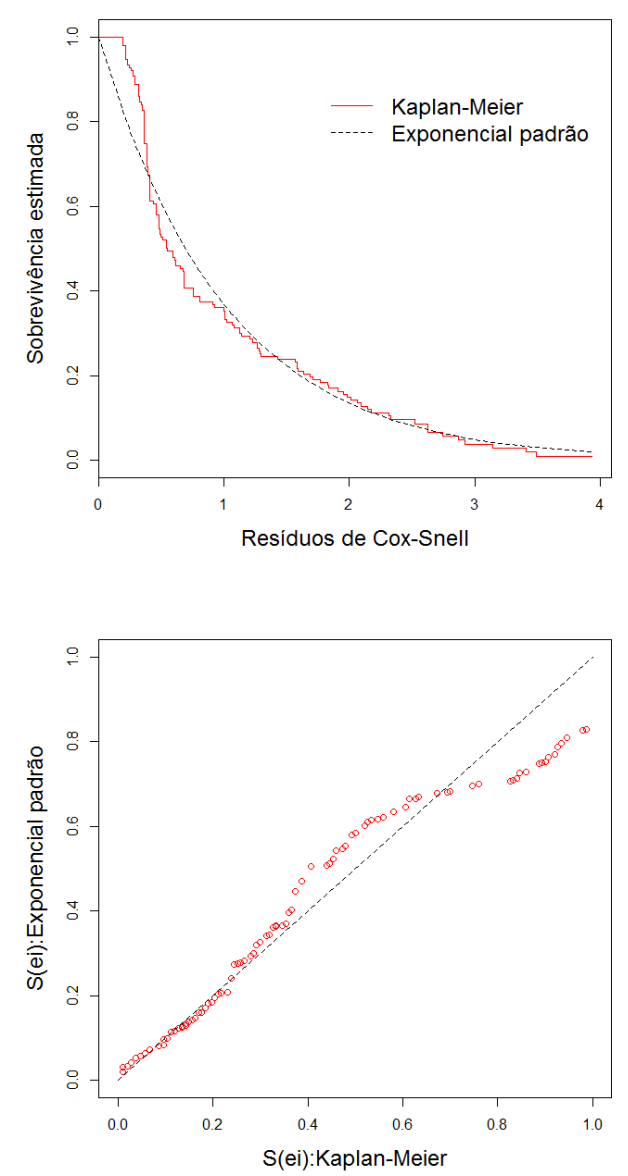

Figura 2- . Comparações das funções de sobrevivência do resíduo de Cox-Snell com a Exponencial padrão

Pode ser observado, a partir da Figura 2, que a função de sobrevivência dos resíduos de Cox-Snell se ajustou bem à função de sobrevivência da exponencial padrão, indicando um bom ajuste do modelo de riscos proporcionais de Cox para os dados dessa aplicação.

\section{Conclusões}

Neste trabalho, o modelo de riscos proporcionais de Cox foi utilizado para ajustar a distribuição do tempo até o alívio da dor em pacientes com dor lombar crônica. A escolha do modelo de Cox neste trabalho foi devido ao fato desse modelo ser um dos mais populares na análise de dados de sobrevivência, principalmente na área de saúde. A principal razão dessa popularidade é a presença da componente não-paramétrica, que torna o modelo bastante flexível. Além disso, a proporcionalidade dos riscos é uma característica muito conveniente, principalmente quando o interesse é interpretar os coeficientes estimados.

A análise contou com 6 variáveis: sexo, idade, IMC, tempo de dor, uso de medicamentos e grupo de tratamento, dos quais somente o grupo de tratamento e uso de medicamentos apresentaram um resultado significativo. 
Além disso, foi verificado que não há multicolinearidade entre as variáveis avaliadas. Os resultados mostraram que os pacientes que foram tratados com corrente interferencial ativa apresentaram menor tempo até o alívio da dor (maior risco de perceber um alívio na dor) quando comparado com um paciente tratado somente com corrente interferencial placebo. Além disso, o uso de medicamentos aumentou o tempo para o alívio da dor. Esses resultados comprovam a efetividade do uso da corrente interferencial no tratamento para pacientes com dor lombar crônica não específica.

Como propostas futuras de trabalho, modelos de regressão paramétricos podem ser considerados como alternativas ao modelo de riscos proporcionais de Cox. Considerando que o número de sessões realizadas é discreto, um modelo discreto deve ser utilizado. Exemplos de trabalhos que consideram a análise de dados discretos de sobrevivência podem ser vistos em Nakano e Carrasco (2006), Carrasco et al. (2012), Brunello e Nakano (2015), entre outros.

\section{Referências}

AIRAKSINEN O.; BROX J. I.; CEDRASCHI C.; HILDEBRANDT J.; KLABER-MOFFETT J.; KOVACS F., et al. Chapter 4. European guidelines for the management of chronic nonspecific low back pain. Eur. Spine J. Suppl 2:S192$300,2006$.

BRUNELLO, G. H. V; NAKANO, E. Y. Inferência bayesiana no modelo weibull discreto em dados com presença de censura. TEMA - Tend. Mat. Apl. Comput., v.16, n.2, p.97-110, 2015.

CARRASCO, C. G.; TUTIA, M. H.; NAKANO, E. Y. Intervalos de confiança para os parâmetros do modelo geométrico com inflação de zeros. TEMA - Tend. Mat. Apl. Comput., v.13, n.3, p.247-255, 2012.

CORRÊA J. B.; COSTA L. O.; OLIVEIRA N. T.; LIMA W. P.; SLUKA K. A.; LIEBANO R. E. Effects of the carrier frequency of interferential current on pain modulation and central hypersensitivity in people with chronic nonspecific low back pain: A randomized placebo-controlled trial. Eur. J. Pain. 2016. [Epub ahead of print]

COSTA L. O.; MAHER C. G.; LATIMER J.; HODGES P. W.; HERBERT R. D.; REFSHAUGE K. M., et al. Motor control exercise for chronic low back pain: a randomized placebo-controlled trial. Phys. Ther, v. 89, n.12, p.1275-86, 2009.

COX, D. R. Regression models and life tables (with discussions). J.R. Stat. Soc. B, v.34, n.2, p.187-202, 1972.

COX, D. R.; SNELL, E. J. A general definition of residuals. J.R. Stat. Soc. B, v.30, n.2, p.248-275, 1968.

DELITTO A; GEORGE S. Z; VAN DILLEN L. R.; WHITMAN J. M.; SOWA G.; SHEKELLE P., et al. Low back pain. J Orthop Sports Phys Ther. v.42, n.4,:A1-57, 2012.

FACCI L. M.; NOWOTNY J. P.; TORMEM F.; TREVISANI V. F. Effects of transcutaneous electrical nerve stimulation (TENS) and interferential currents (IFC) in patients with nonspecific chronic low back pain: randomized clinical trial. Sao Paulo Med. J. v.129, n.4:206-16, 2011.

FUENTES J. P, ARMIJO OLIVO S., MAGEE D. J., GROSS D. P. Effectiveness of interferential current therapy in the management of musculoskeletal pain: a systematic review and meta-analysis. Phys. Ther. v.90, n.9, p.1219-38, 2010 .

GRAMBSCH, P. M.; THERNEAU, T. M. Proportional hazards tests and diagnostics based on weighted residuals. Biometrika, v.81, n.3, p.515-526, 1994.

KAPLAN, E. L.; MEIER, P. Nonparametric estimation from incomplete observations. J. Am. Stat. Assoc., v.53, n.282, p.457-481, 1958.

LAWLESS, J. F. Statistical models and methods for lifetime data. 2.ed. New Jersey: John Wiley \& Sons, 2003. 
MAIA, M. A.; NAKANO, E. Y. Análise do tempo até a re-hospitalização de pacientes com esquizofrenia via modelo de riscos proporcionais de Cox. Semina: Ciências Exatas e Tecnológicas, v.37, n.2, p.51-58, 2016.

NAKANO, E. Y.; CARRASCO, C. G. Uma avaliação do uso de um modelo contínuo na análise de dados discretos de sobrevivência. TEMA - Tend. Mat. Apl. Comput., v.7, n.1, p.91-100, 2006.

NAKANO, E. Y.; CUNHA, J. F. Análise do efeito da camuflagem no tempo de segregação em regiões texturizadas utilizando o modelo de riscos proporcionais de Cox. Semina: Ciências Exatas e Tecnológicas, v.33, n.2, p.141-148, 2012.

NETER, J.; WASSERMAN, W.; KUTNER, M. H. Applied Linear Regression Models. 3.ed, Homewood, IL: Irwin, 1990.

OSTELO, R. W.; de VET, H. C. Clinically important outcomes in low back pain. Best Pract Res Clin Rheumatol., v.19, n.4, p.593-607, 2005.

R CORE TEAM. R: A language and environment for statistical computing. R Foundation for Statistical Computing, Vienna, Austria. ISBN 3-900051-07-0, URL http://www.R-project.org, 2016.

SANTOS, R. O; NAKANO, E. Y. Análise do tempo de permanência de trabalhadores no mercado de trabalho do Distrito Federal via modelo de riscos proporcionais de Cox e Log-normal. Rev. Bras. Biom., v.33, n.4, p.570-584, 2015.

SCHOENFELD, D. Partial Residuals for the proportional hazard regression model. Biometrika, v.69, n.1, p.329$241,1982$.

TULDER, M. V. European guidelines for the management of chronic nonspecific low back pain. Eur. Spine J. (15(Suppl 2)):134-35, 2006. 